Article

\title{
Optimal Disintegration Strategy in Multiplex Networks under Layer Node-Based Attack
}

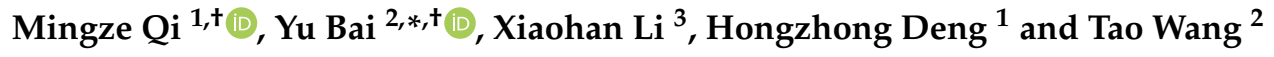 \\ 1 College of Systems Engineering, National University of Defense Technology, \\ Changsha 410073, Hunan, China; qimingze17@nudt.edu.cn (M.Q.); denghongzhong@nudt.edu.cn (H.D.) \\ 2 College of Computer, National University of Defense Technology, Changsha 410073, Hunan, China; \\ taowang2005@nudt.edu.cn \\ 3 School of Mathematics, Shandong University, Jinan 250100, Shandong, China; LiXiaohan23@163.com \\ * Correspondence: baiyu09a@nudt.edu.cn \\ + These authors contributed equally to this work.
}

Received: 28 August 2019; Accepted: 18 September 2019; Published: 21 September 2019

\begin{abstract}
From social networks to complex infrastructures, many systems could be described by multiplex networks formed by a set of nodes connected via more than one type of links. Network disintegration, which is the problem of identifying a set of nodes or edges whose removal would maximize the network collapse, is significant for dismantling harmful networks. In this article, we consider the optimal disintegration strategy problem in multiplex networks and extend the attack mode to the layer node-based attack. An optimization model is proposed to search the optimal strategy of a multiplex network under layer node-based attack with fix attack length. Two types of strategies based on the information of multiplex nodes and layer nodes, respectively, are also given for comparison. Through experiments in both model networks and real networks, we found that the approximate optimal strategies could be identified by solving the model. The properties of the optimal strategies are also summarized.
\end{abstract}

Keywords: network robustness; network disintegration; multiplex network; layer node-based attack

\section{Introduction}

Recent decades have witnessed a dramatic advance in network sciences [1]. Network robustness, as an important field of complex networks, aims to improve the resilience of systems and to construct more efficient architecture [2-4]. However, not all networks are beneficial, some of them are harmful to human life, for instance, the epidemic or rumor spreading networks [5,6]. For these networks, we need to break its functionality by removing some nodes or links. This is the focus of this paper, namely, network disintegration problem, which is also called network dismantling or network attack $[7,8]$.

Over the past few decades, there have been a large variety of works focusing on this problem. The most traditional solutions are nodes-based methods, where the nodes are removed in decreasing order of their significance determined by some indices, such as degree centrality [9], betweenness centrality [10], and others [11,12]. Recent years, many algorithms are presented to identify the minimal nodes set whose removal would collapse the giant connected component. Examples include collective influence algorithm [13], CoreHD algorithm [14], Explosive immunization algorithm [15], Min-sum algorithm [7], GND algorithm [16] and so on. In this article, we consider a more generalized problem, named the disintegration strategy problem, where we consider limited attack resource and aim to maximize the disintegration effect under fixed attack length. The methods mentioned above could also be used to develop disintegration strategies when we remove a specified number of nodes in the given order. Moreover, for searching the optimal disintegration strategy, there have been 
some combinatorial optimization-based studies in undirected [17] and directed [18] networks. In these studies, some meta-heuristic algorithms were introduced to identify the optimal disintegration strategy.

Considering that networks rarely exist independently, the research of network science was extended to a multilayer framework [19]. According to the difference between the types of nodes in each layer and the links between the nodes in different layers [20], multilayer networks could be divided into multiplex networks, interdependent networks and so on [21]. Especially, the multiplex network could be regarded as a set of nodes connected by different types of links. Examples include the individuals connected through various social relationships [22] and the cities linked by different transportation networks [23]. The study of multiplex networks has emerged as one of the significant topics in network science and recent research proved that the co-existence of different interactions in multiplex networks would strongly affect the properties of the systems [24].

Therefore, the network disintegration problem was also generalized to multiplex networks. Saeed et al. [25] firstly extended the existing disintegration strategies to multiplex networks, and pointed out that ignoring the multi-layer structure of the systems will cause the result of overestimating the robustness of them. G. J. Baxter et al. [26] proposed a heuristic strategy named Effective Multiplex Degree for dismantling interdependent or multiplex networks. The meta-heuristic algorithm has also been introduced to determine the optimal disintegration strategy in general multiplex networks [27].

Nevertheless, an important hypothesis in the above studies is that the nodes in each layer would fail after being attacked. Actually, in some realistic cases, the targets that are attacked or those which fail may be just the layer nodes [28]. For instance, in the multiplex social networks, the users are banned from using one or some, but not all social network sites. Correspondingly, in the multiplex transport networks, different traffic stations in a city, like airports or highways, may not fail at the same time. We name this kind of attack as the layer node-based attack.

In this paper, we focus on the disintegration strategy problem in multiplex networks. The optimization model of disintegration strategy in multiplex networks is improved to adapt the layer node-based attack. In artificial coupled networks with different degree correlated and many real networks, we compare our algorithm with some existing methods.

\section{Optimization Model of Disintegration Strategy in Multiplex Networks under Layer Node-Based Attack}

In this section, we extend the optimization model of the disintegration strategy in the multiplex network to different attack modes, multiplex node-based attack and layer node-based attack, of which we give an example in Figure 1. The removal of individuals means the removal of all kinds of edges connecting them and is named multiplex node-based attack [28]. Moreover, the network could also be embedded in the framework of the multiplex network with two layers, which are given in Figure $1 \mathrm{c}, \mathrm{d}$. The layer node-based attack is the attack of nodes in one layer, which means the removal of all edges connecting the nodes in this layer, and the edges in other layers are not affected.

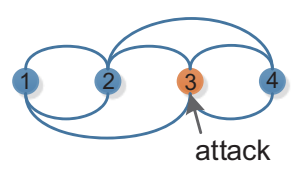

(a)

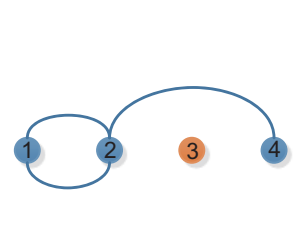

(b)

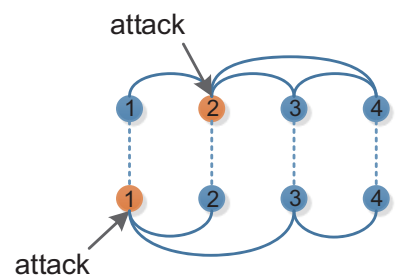

(c)

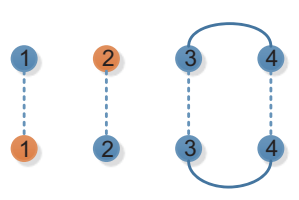

(d)

Figure 1. Multiplex node-based attack (a) and layer node-based attack (c) in the illustrative examples of the multiplex network. The edges in the top and bottom represent different kinds of edges. The two networks after different attacks are given in $(\mathbf{b}, \mathbf{d})$. 
We consider a multiplex network composed of $N$ individuals (multiplex nodes) and $M$ layers in which edges are unweighted and undirected. It could be formed as a tuple $G=(V, E)$, where $V(G)$ is the set of multiplex nodes and $E(G)$ is the set of edges connecting these multiplex nodes in different layers. Each multiplex node $v_{i},(i=1, \ldots, N)$ in $V$ could be represented by a set of layer nodes $\left[v_{i}^{1}, \ldots, v_{i}^{M}\right]$. Each layer $\alpha$ is presented by an adjacency matrix $A^{\alpha}=\left\{a_{i j}^{\alpha}\right\}_{N \times N}$, where $a_{i j}^{\alpha}=1$ if layer nodes $v_{i}^{\alpha}$ and $v_{j}^{\alpha}$ are connected through an edge (i.e., multiplex nodes $v_{i}$ and $v_{j}$ are connected through an edge on layer $\alpha$ ). The multiplex network could be completely determined by the vector of the adjacency matrixes of the $\mathrm{M}$ layers

$$
\boldsymbol{A}=\left\{A^{1}, \ldots, A^{M}\right\}
$$

We define the degree of a layer node $v_{i}^{\alpha}$ (or the layer degree of a multiplex node $v_{i}$ on layer $\alpha$ ) as $k_{i}^{\alpha}=\sum_{j} a_{i j}^{\alpha}$, which is the number of adjacent edges of layer node $v_{i}^{\alpha}$, and the layer degree of the multiplex node $v_{i}$ in all $M$ layer is the vector

$$
k_{i}=\left\{k_{i}^{1}, \ldots, k_{i}^{M}\right\}
$$

Corresponding, we define the multiplex degree of multiplex node $v_{i}$ as $k_{i}=\sum_{\alpha} k_{i}^{\alpha}$, which is the total number of adjacent edges of multiplex node $v_{i}$ in all layers.

Moreover, we define the aggregated topological adjacency matrix $\mathcal{A}=\left\{a_{i j}\right\}_{N \times N}$ of a multiplex network, where

$$
a_{i j}=\left\{\begin{array}{c}
1, \text { if } \exists \alpha: a_{i j}^{\alpha}=1 \\
0, \text { otherwise }
\end{array}, i=1, \ldots, N ; j=1, \ldots, N\right.
$$

This is also the adjacency matrix of an unweighted network obtained from the multiplex network, which is named aggregated network. The nodes $v_{i}$ and $v_{j}$ in the aggregated network are connected when there are edges between them in at least one layer of the multiplex network.

In this article, the disintegration approaches focus on the layer node removal, and we assume that if a layer node is removed, the attached edges of it in this layer are removed. Meanwhile, we assume that all the layers in the multiplex network are coupled in a connective/complementary manner. That is to say, the removal of edges in one layer does not affect the layer nodes and edges in other layers and the function of the edges could be replaced by the edges in other layers. Examples include multiplex transportation networks composed of different modes of transportation and multiplex online social networks composed of various social media.

Denote by $\widehat{V} \subseteq V$ the set of layer nodes that are removed and denote by $\widehat{G}=(V-\widehat{V}, \widehat{E})$ the network after the nodes are removed. Denote by $n=|\widehat{V}|$ the disintegration strength parameter and $q=n /(N \times M)$ is the removed ratio of the nodes. We define a disintegration strategy as

$$
\widehat{X}=\left[\begin{array}{cccc}
x_{11} & x_{12} & \cdots & x_{1 N} \\
x_{21} & x_{22} & \cdots & x_{2 N} \\
\vdots & \vdots & \ddots & \vdots \\
x_{M 1} & x_{M 2} & \cdots & x_{M N}
\end{array}\right]
$$

where $x_{\alpha i}=1$ if layer node $v_{i}^{\alpha} \in \widehat{V}$, otherwise $x_{\alpha i}=0$. It is easy to obtain that

$$
n=\sum_{\alpha=1}^{M} \sum_{i=1}^{N} x_{\alpha i}
$$

The goal of the optimization model is to identify the optimal solution $\widehat{X}^{*}$ that could maximize the disintegration effect. The optimization model for the disintegration strategy in a multiplex network under layer node-based attack can be described as follows: 


$$
\text { s.t. }\left\{\begin{array}{c}
\max \Phi(\widehat{X}) \\
\sum_{\alpha=1}^{M} \sum_{i=1}^{N} x_{\alpha i}=n \\
x_{\alpha i}=0 \text { or } 1, \alpha=1, \ldots, M ; i=1,2, \ldots, N
\end{array}\right.
$$

Considering the complementary relationship of different layers in the multiplex network, we use the size of the giant component $C$ in the aggregated network as the measure function of the network performance. The $\Phi(\widehat{X})=C(G)-C(\widehat{G})$ is used to measure the disintegration effect of strategy $\widehat{X}$. For a network with $N$ multiplex nodes and $M$ layers, there are $C_{N M}^{n}$ ways to pick $n$ nodes for removal, which increases sharply with $N$ and $M$. Therefore, we consider solving this problem using some meta-heuristic algorithms. In particular, the tabu search algorithm has been proved to be an effective method for solving similar problems in single-layer networks [17]. In this article, we similarly use the tabu search (TS) algorithm to seek the optimal solution of the above optimization model. The details of the TS algorithm could be found in references $[17,29,30]$. In the experiments of this article, we set the maximum of iterations $T_{\max }=1000$, the number of candidates $n_{c a n}=100$ and the length of the tabu list $L=50$. We could obtain the approximate optimal disintegration strategy by solving the optimization model in Formula (6) through the TS algorithm.

\section{Experimental Analysis}

In this section, we will verify the effect of the optimal disintegration strategies in both synthetic and real networks. For comparison, we introduce some existing disintegration strategies which are generalized from single-layer disintegration schemes.

\subsection{The Existing Disintegration Strategies}

There have been many disintegration strategies in monoplex networks. We extend some of them to the layer node-based attack in multiplex networks. Considering the particularity of multiplex networks, we divide the strategies into two categories according to the nodes information we used: multiplex node-based strategies and layer node-based strategies. The high multiplex degree (HMD) strategy, the high multiplex degree adaptive (HMDA) and the effective multiplex degree (EMD) strategy are based on the information of the multiplex nodes in networks and are called multiplex node-based strategies. The high layer degree (HLD) strategy, the high layer degree adaptive (HLDA) strategy and the collective influence (CI) strategy are based on the information of the layer nodes in networks and name layer node-based strategies.

\subsubsection{Multiplex Node-Based Strategies}

For multiplex node-based strategies, we order the multiplex nodes according to the global information of the multiplex network and remove the layer nodes included by the multiplex nodes in each layer consecutively. The high degree strategy is the easiest method for disintegrating a network. We remove the layer nodes successively according to the multiplex degree of the multiplex nodes they belong to and name this method as high multiplex degree (HMD) strategy. If we recalculate the multiplex degree after removing all the layer nodes of the multiplex node with the highest multiplex degree at each step, this is referred to the high multiplex degree adaptive (HMDA) strategy.

Another strategy is a heuristic strategy named effective multiplex degree (EMD) [26], which is designed for attacking interdependent networks. The weight of the multiplex nodes $w_{i}$ are calculated iteratively according to the following formula:

$$
w_{i}^{(t+1)}=\sum_{l=1}^{M} \sum_{j \in \mathcal{N}^{\alpha}} \frac{1}{M_{j}} \frac{w_{j}^{(t)}}{k_{j}^{(\alpha)}}
$$


where $\mathcal{N}^{\alpha}$ is the set of neighbors of multiplex node $v_{i}$ in layer $\alpha$ and $k_{j}^{(\alpha)}$ is the layer degree of multiplex node $v_{j}$ on layer $\alpha, M_{j}$ is the number of layers in which multiplex node $v_{j}$ participates. We make all the $M_{j}=M$ in this paper and the initial value of the $w_{i}$ is the multiplex degree $k_{i}$ of node $v_{i}$. It is worth noting that the EMD strategy is also "adaptive", meaning that we recalculate all the weights of the remaining multiplex nodes after removing all the layer nodes of a multiplex node. The iterations $t$ in each calculation is 10 .

\subsubsection{Layer Node-Based Strategies}

In layer node-based strategies, we calculate the scores of layer nodes in each layer respectively and order all the layer nodes in all layers. Similarly, high layer degree (HLD) is a strategy where we remove the layer nodes according to their layer degree. The high layer degree adaptive (HLDA) strategy is also defined by the adaptive computation of the layer degree.

Meanwhile, we introduce collective influence (CI) strategy to the layer node based attack [13]. The CI strength of a layer node $v_{i}^{\alpha}$ is defined as:

$$
C I_{l}(i)=\left[k_{i}^{\alpha}-1\right] \sum_{j \in \partial \operatorname{Ball}(i, l)}\left[k_{j}^{\alpha}-1\right]
$$

where $\partial B$ all $(i, l)$ denotes the set formed by all the layer nodes in layer $\alpha$ that are at distance $l$ to node $v_{i}^{\alpha}$. The integer $l$ is an adjustable parameter and we set $l=2$ in this paper. In each step, we also need to iteratively calculate the $\mathrm{CI}$ length of all the layer nodes and remove the one with the highest CI length.

\subsection{Experiments in Synthetic Networks with Different Degree Correlation}

Firstly, we consider the synthetic networks obtained from the Erdős-Rényi (ER) model in which the $N$ nodes are connected randomly with probability $p$. Meanwhile, previous research has proven that the interlayer degree correlation has a great impact on the response to the attack [31]. Therefore, we consider different multiplex networks including two layers coupled with three correlated structure: maximally negative (MN), uncorrelated (UC), and maximally positive (MP). In the $\mathrm{MN}$ case, the degree of nodes in different layers are maximally anti-correlated in their degree order, while they are maximally correlated in the MP case. For better understanding, we give a schematic illustration in Figure 2.

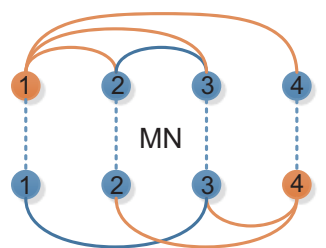

(a)

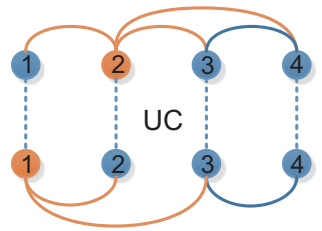

(b)

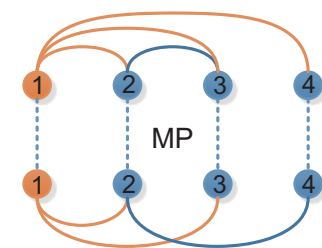

(c)

Figure 2. Schematic illustration of three kinds of correlated structure, maximally negative (a), uncorrelated (b) and maximally positive (c). The nodes and edges that are failure under HLD strategy are shown in orange.

The results of the optimal disintegration strategy and the six existing strategies are given in Figure 3. For better demonstration, we use dotted lines and solid lines to represent the multiplex node-based strategies and layer node-based strategies, respectively. Meanwhile, strategies with the same principle (like HMD and HLD strategies) are in the same color.

The results in Figure 3 show that for the strategies with the same principle, the layer node-based strategies generally have better performance than the multiplex node-based strategies. The advantage decreases as the degree of different layer nodes tend to be positively correlated. Meanwhile, the EMD strategy has poor performance compared to the general HMD strategy when there is no interdependent 
relation between different layers. In particular, we find that the effect of the HLD and CI strategy approaches that of optimal disintegration strategy when the network is on the brink of collapse.

(a)

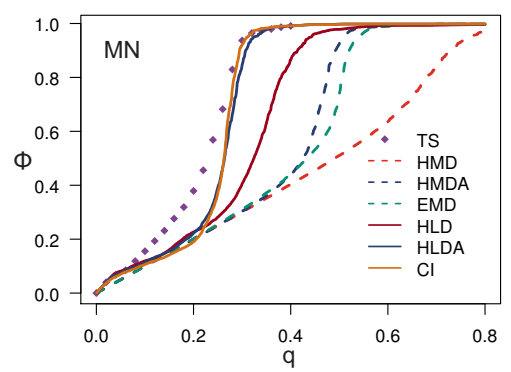

(b)

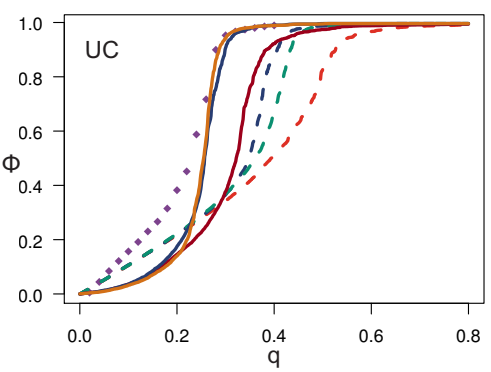

(c)

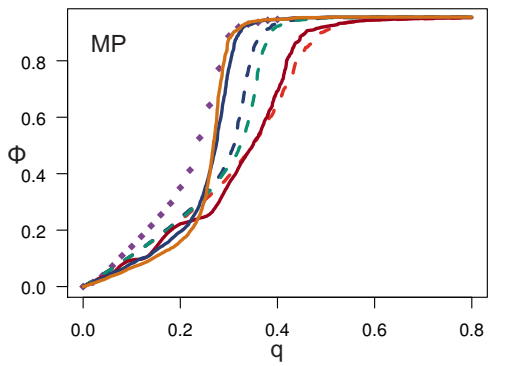

Figure 3. Optimal disintegration strategies in coupled ER networks with different degree correlation under layer node-based attack. The two layers are obtained independently with probability $p=0.006$ and size $N=500$. The two layers are coupled with there correlated structure (MN, UC and MP), in figures (a-c) respectively. We determine the mean value of $\Phi$ over 20 independent realizations.

\subsection{Experiments in Real Networks}

There is a large difference between the model networks and real systems in the edge connection and interlayer degree correlation. Therefore, in this section, we introduce some real multiplex networks for experiments.

1. Caenorhabditis elegans connectome, where the multiplex consists of layers corresponding to different synaptic junctions: electric (Electric), chemical monadic (Chem. Mon), and polyadic (Chem. Pol). We use different pairs of layers to construct multiplex networks and obtain three different duplex networks.

2. Drosophila melanogaster genetic and protein interaction network, where the multiplex consists of layers corresponding to different types of genetic interactions for organisms: direct interaction (Direct), suppressive genetic interaction (Supp. Gen) and additive genetic interaction (Add. Gen). We similarly use different pairs of layers to construct multiplex networks.

3. Top Noordin network, where the multiplex consists of three layers corresponding to different relationships among extremists active in Indonesia: trust $(\mathrm{T})$, operations $(\mathrm{O})$ and communication (C).

More information about these networks is given in Table 1.

Table 1. The basic information of the real multiplex networks. The first column represents the names of the different multiplex networks and the second column identifies the names of different layers used to construct multiplex networks. The other columns contain information about the number of nodes $N$, the total number of edges in the aggregated network of each multiplex network $E^{[A]}$ and the number of edges in each layer $\left(E^{[1]}, E^{[2]}\right.$ and $\left.E^{[3]}\right)$.

\begin{tabular}{ccccccc}
\hline Network & Layers & $N$ & $\boldsymbol{E}^{[A]}$ & $\boldsymbol{E}^{[\mathbf{1}]}$ & $\boldsymbol{E}^{[\mathbf{2}]}$ & $\boldsymbol{E}^{[\mathbf{3}]}$ \\
\hline \multirow{3}{*}{ Caenorhabditis Elegans } & Electric - Chem. Mon. & 238 & 1833 & 1576 & 887 & $/$ \\
& Electric-Chem. Pol & 252 & 1147 & 485 & 773 & $/$ \\
& Chem. Mon.-Chem. Pol & 259 & 1804 & 511 & 1455 & $/$ \\
\hline \multirow{3}{*}{ Drosophila Melanogaster } & Direct - Supp. Gen. & 676 & 1946 & 668 & 1344 & $/$ \\
& Direct-Add. Gen. & 625 & 1498 & 523 & 1024 & $/$ \\
& Supp. Gen.-Add. Gen. & 557 & 2117 & 1421 & 1164 & $/$ \\
\hline Top Noordin Extermist & T-O-C & 79 & 620 & 259 & 437 & 200 \\
\hline
\end{tabular}

The results of the six duplex networks under different disintegration strategies are given in Figures 4 and 5 . 
(a) Electric - Chem. Mon.

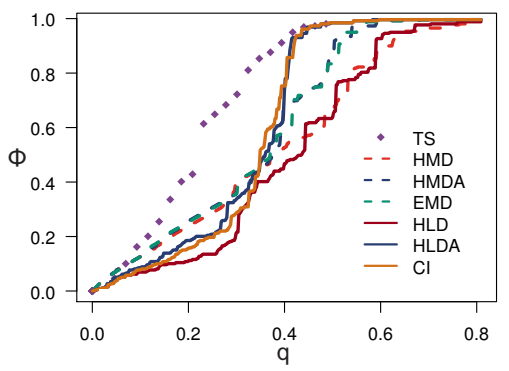

(b) Electric - Chem. Pol

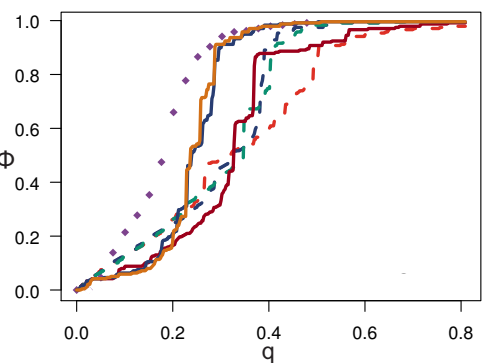

(c) Chem. Mon. - Chem. Pol

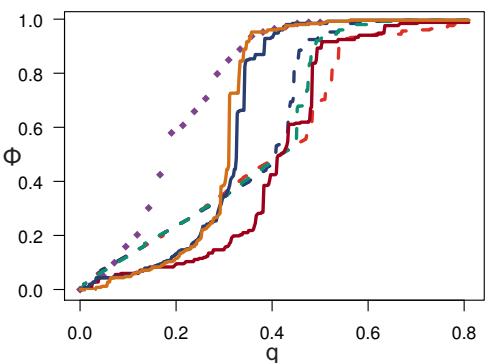

Figure 4. Optimal disintegration strategies in multiplex Caenorhabditis Melanogaster neural networks under layer node-based attack. For the TS algorithm, we use the maximum value of $\Phi$ in 20 independent realizations.

(a) Direct - Supp. Gen

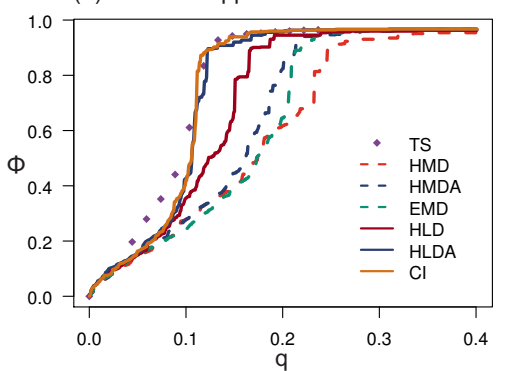

(b) Direct - Add. Gen

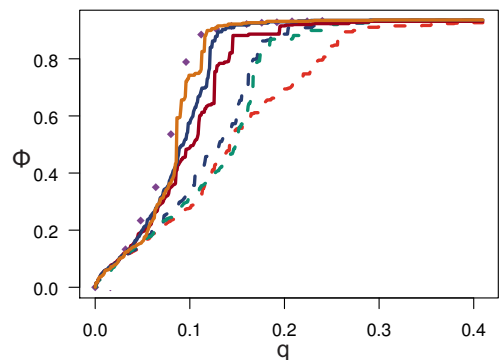

(c) Supp. Gen. - Add. Gen.

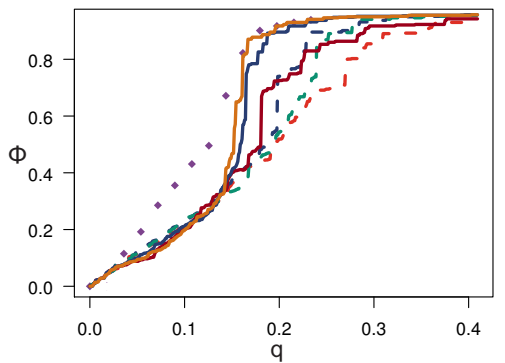

Figure 5. Optimal disintegration strategies in multiplex Drosophila Melanogaster genetic and protein interaction networks under layer node-based attack. For the TS algorithm, we use the maximum value of $\Phi$ in 20 independent realizations.

Some conclusions obtained from Figure 3 are also shown in Figures 4 and 5. The performance of HLDA and CI strategies are generally better than the other strategies and close to that of optimal strategy when the network is close to collapse. Differently, for multiplex networks with a higher average degree in Figure 4, the performance of the layer node-based strategies is better than that of multiplex node-based strategies when the percentage of node removed is small (less than 0.2) and the optimal strategies have more advantages than other existing strategies. However, for the relatively sparse multiplex networks in Figure 5, some layer node-based strategies have similar performance with the optimal strategies and general advantage through multiplex node-based strategies in different attack length.

We further experiment in the top Noordin extremist multiplex network with three layers. To get a better disintegration effect, we use the best solution obtained from the last search as the initial solution for the next search (i.e., we randomly add an extra node in the optimal strategy with attack length $n$ and use it as the initial solution when searching the optimal strategy with attack length $n+1$ ). The results of the optimal disintegration strategies and other strategies are presented in Figure 6. To better display the properties of the optimal strategy, we give the visualization of the nodes included in the optimal disintegration strategy and the aggregated network of the multiplex network after nodes removed on the left side of the graph. Meanwhile, on the right side, we give a visualization of the strategy given by HLDA algorithm with the same removal ratio of nodes where the optimal disintegration strategy has the greatest advantage over the other strategies.

The results in Figure 6 show that the optimal disintegration strategies outperform the other existing strategies and the effect of HLDA and CI strategies are also close to that of the optimal strategy when the network is about to collapse. By the visualization on both sides of Figure 6, we find that the two strategies have a large overlapping proportion in some layer nodes with high degree. Differently, the removal of nodes in optimal disintegration strategy divides the aggregated network into many clusters which disconnect to each other. However, in the layer node-based strategy like HLDA, though some "hub" layer nodes are removed, the corresponding multiplex nodes could still be 
connected through the backup edges in the other layer, and in the multiplex node-based strategies, we preferentially remove the layer nodes of the "hub" multiplex nodes in all layers. So the effect of multiplex node-based strategies is better than that of layer node-based strategies when only a small percentage of layer nodes are removed. Since backup edges are on different layers, the multiplex network can only crash if all the layers are crashed. Therefore, some methods based on adaptive layer nodes, such as CI or HLDA, will be effective and have a similar effect with the optimal strategy when the network is close to collapse.

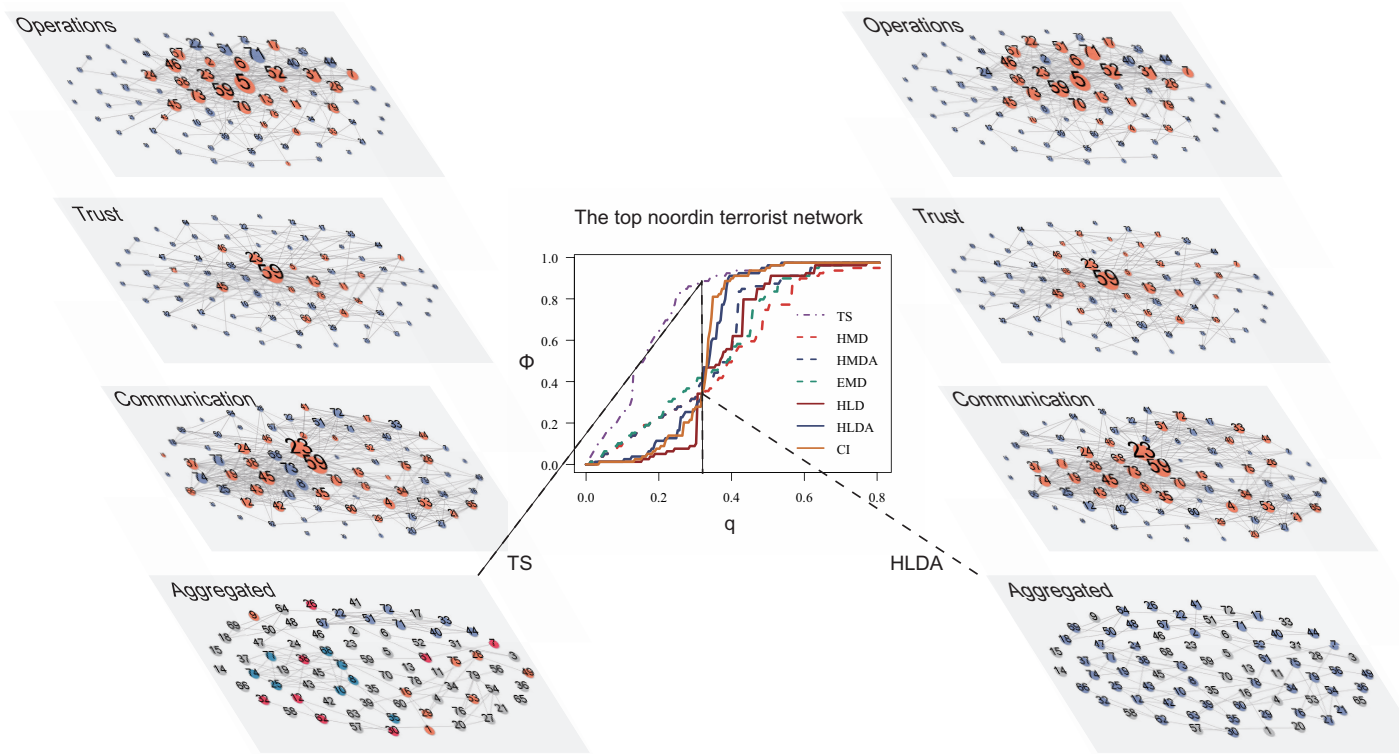

Figure 6. Optimal disintegration strategies in the three-layer top Noordin extremist network under layer node-based attack. For the TS algorithm, use the maximum value of $\Phi$ in 20 independent realizations. The left side of the figure shows the layer nodes (the orange nodes in the first three layers) included in the optimal disintegration strategy when the percentage of node removed is about 0.32 (where $\Phi=0.886$ ). The size of the nodes is determined by their layer degree. At the bottom, we give the aggregated network of the multiplex network after nodes removed in the fourth layer, where four giant components with size 8 are in different colors. Similarly, we present a visualization of the HLDA strategy on the right side of the figure (where $\Phi=0.316$ and the size of the giant component in the aggregated network is 51).

\section{Conclusions and Discussion}

In this article, we extend the optimization model of the disintegration strategy in the multiplex network to layer node-based attack mode. The tabu search algorithm is introduced to solve the optimization model. Experiments in the model and real multiplex networks prove that the approximate optimal disintegration strategies obtained from the TS algorithm are superior to other strategies in different attack length. The optimal strategies divide the aggregated network of the multiplex networks into several independent clusters by removing some layer nodes from different layers. The advantages of them are especially obvious in the denser networks and shorter attack length. Meanwhile, we found that the effect of the optimal disintegration strategy is close to that of some layer node-based strategies when we need to make the multiplex networks complete collapse.

This article focuses on the general multiplex network where different layers are independent and share a set of nodes. Actually, there are many other coupling ways between networks in reality, such as interdependence or collaboration. The same problem in these networks would be the future research direction. 
Author Contributions: Conceptualization, M.Q. and Y.B.; methodology, M.Q., Y.B. and X.L.; software, M.Q., Y.B. and X.L.; validation, H.D. and T.W.; formal analysis, M.Q. and Y.B.; investigation, M.Q. and Y.B.; resources, H.D.; data curation, M.Q.; visualization, M.Q. and Y.B.; supervision, H.D. and T.W.

Funding: This work was supported by the National Key R\&D Program of China [2018YFB1003903] and the National Natural Science Foundation of China (NSFC) [grant number 71771214, 71690233, 71971213].

Acknowledgments: The authors are grateful to Ye Deng and Ziqiang Cao for their help on this paper.

Conflicts of Interest: The authors declare no conflict of interest.

\section{References}

1. Newman, M. Networks: An Introduction. Astronomische Nachrichten 2010, 327, 741-743.

2. Bollobás, B.; Riordan, O. Robustness and Vulnerability of Scale-Free Random Graphs. Internet Math. 2004, 1, 1-35. [CrossRef]

3. Wu, J.; Barahona, M.; Tan, Y.J.; Deng, H.Z. Spectral Measure of Structural Robustness in Complex Networks. Syst. Man Cybern. 2011, 41, 1244-1252. [CrossRef]

4. Zhang, G.; Yu, S.S.; Zou, S.; Iu, H.H.C.; Fernando, T.; Zhang, Y. An Investigation into Cascading Failure in Large-Scale Electric Grids: A Load-Redistribution Approach. Appl. Sci. 2018, 8, 1033. [CrossRef]

5. Moreno, Y.; Nekovee, M.; Pacheco, A.F. Dynamics of rumor spreading in complex networks. Phys. Rev. E 2004, 69, 66130. [CrossRef] [PubMed]

6. Pastor-Satorras, R.; Castellano, C.; Mieghem, P.V.; Vespignani, A. Epidemic processes in complex networks. Rev. Mod. Phys. 2015, 87, 925-979. [CrossRef]

7. Braunstein, A.; Dall'Asta, L.; Semerjian, G.; Zdeborová, L. Network dismantling. Proc. Natl. Acad. Sci. USA 2016, 113, 12368-12373. [CrossRef] [PubMed]

8. Mugisha, S.; Zhou, H.J. Identifying optimal targets of network attack by belief propagation. Phys. Rev. E 2016, 94, 12305. [CrossRef] [PubMed]

9. Albert, R.; Jeong, H.; Barabási, A.L. Error and attack tolerance of complex networks. Nature 2000, 406, 378-382. [CrossRef] [PubMed]

10. Brandes, U. A Faster Algorithm for Betweenness Centrality. J. Math. Sociol. 2001, 25, 163-177. [CrossRef]

11. Lü, L.; Chen, D.; Ren, X.L.; Zhang, Q.M.; Zhang, Y.C.; Zhou, T. Vital nodes identification in complex networks. Phys. Rep. 2016, 650,1-63. [CrossRef]

12. Jiang, L.; Jing, Y.; Hu, S.; Ge, B.; Xiao, W. Identifying Node Importance in a Complex Network Based on Node Bridging Feature. Appl. Sci. 2018, 8, 1914. [CrossRef]

13. Morone, F.; Makse, H.A. Influence maximization in complex networks through optimal percolation. Nature 2015, 524, 65-68. [CrossRef] [PubMed]

14. Zdeborová, L.; Zhang, P.; Zhou, H.J. Fast and simple decycling and dismantling of networks. Sci. Rep. 2016, 6, 37954. [CrossRef] [PubMed]

15. Clusella, P.; Grassberger, P.; Perez-Reche, F.J.; Politi, A. Immunization and Targeted Destruction of Networks using Explosive Percolation. Phys. Rev. Lett. 2016, 117, 208301. [CrossRef] [PubMed]

16. Ren, X.L.; Gleinig, N.; Helbing, D.; Antulov-Fantulin, N. Generalized network dismantling. Proc. Natl. Acad. Sci. USA 2019, 116, 6554-6559. [CrossRef] [PubMed]

17. Deng, Y.; Wu, J.; Tan, Y. Optimal attack strategy of complex networks based on tabu search. Phys. A-Stat. Mech. Its Appl. 2016, 442, 74-81. [CrossRef]

18. Yu, Y.; Deng, Y.; Tan, S.; Wu, J. Efficient disintegration strategy in directed networks based on tabu search. Phys. A-Stat. Mech. Its Appl. 2018, 507, 435-442. [CrossRef]

19. Buldyrev, S.V.; Parshani, R.; Paul, G.; Stanley, H.E.; Havlin, S. Catastrophic cascade of failures in interdependent networks. Nature 2010, 464, 1025-1028. [CrossRef]

20. Wang, Z.; Wang, L.; Szolnoki, A.; Perc, M. Evolutionary games on multilayer networks: A colloquium. Eur. Phys. J. B 2015, 88, 124. [CrossRef]

21. Lee, K.M.; Min, B.; Goh, K.I. Towards real-world complexity: An introduction to multiplex networks. Eur. Phys. J. B 2015, 88, 48. [CrossRef]

22. Szell, M.; Thurner, S. Measuring social dynamics in a massive multiplayer online game. Soc. Netw. 2010, 32, 313-329. [CrossRef] 
23. Banavar, J.R.; Maritan, A.; Rinaldo, A. Size and form in efficient transportation networks. Nature 1999, 399, 130-132. [CrossRef] [PubMed]

24. Granell, C.; Gomez, S.; Arenas, A. Dynamical Interplay between Awareness and Epidemic Spreading in Multiplex Networks. Phys. Rev. Lett. 2013, 111, 128701. [CrossRef] [PubMed]

25. Osat, S.; Faqeeh, A.; Radicchi, F. Optimal percolation on multiplex networks. Nat. Commun. 2017, 8, 1540. [CrossRef] [PubMed]

26. Baxter, G.J.; Timár, G.; Mendes, J.F.F. Targeted damage to interdependent networks. Phys. Rev. E 2018, 98, 032307. [CrossRef]

27. Qi, M.; Deng, Y.; Deng, H.; Wu, J. Optimal disintegration strategy in multiplex networks. Chaos 2018, 28, 121104. [CrossRef]

28. Zhao, D.; Wang, L.; Zhi, Y.; Zhang, J.; Wang, Z. The robustness of multiplex networks under layer node-based attack. Sci. Rep. 2016, 6, 24304-24304. [CrossRef]

29. Glover, F.W. Tabu Search-Part I. Orsa J. Comput. 1989, 1, 89-98. [CrossRef]

30. Glover, F.W. Tabu Search-Part II. Informs J. Comput. 1989, 2, 4-32. [CrossRef]

31. Min, B.; Yi, S.D.; Lee, K.M.; Goh, K.I. Network robustness of multiplex networks with interlayer degree correlations. Phys. Rev. E 2014, 89, 42811. [CrossRef] [PubMed]

(C) 2019 by the authors. Licensee MDPI, Basel, Switzerland. This article is an open access article distributed under the terms and conditions of the Creative Commons Attribution (CC BY) license (http:/ / creativecommons.org/licenses/by/4.0/). 